\title{
How Effectively Support Adults with Low Basic Skills? Engagement of Research into Support Schemes Targeted at Adults with Low Basic Skills
}

\author{
Jadwiga Fila1, Agnieszka Rybińska ${ }^{2}$ \\ ${ }^{1,2}$ Foundation for the Development of the Education System, Warsaw, Poland
}

\section{ABSTRACT}

Although the proportion of tertiary-educated adults doubled in Poland in the last 30 years, Poland still lacks a strong culture of adult learning. According to PIAAC research, the percentage of adults with low foundational skills (both literacy and numeracy) reaches the level of $20 \%$. Even more of them have problems with ICT-skills. In comparison to other EU countries, the willingness to participate in different forms of education among Polish adults is quite low. The highest number of people with low basic skills is among those professionally inactive and/or long-term unemployed. Those people are very often equally socially excluded and are suffering from learned helplessness which makes involving them in any educational project very challenging.

Following the guidelines of the Council of Europe on Upskilling pathways: new Opportunities for Adults, in Poland was launched the project "Chances - new opportunities for adults". Its objective was to create and test in practice innovative models of effective educational support for adults with low basic skills. First step of the project was a research scheme, which included desk research, study visits and qualitative research (IDIs) in 50 organizations actively helping adults developing their foundational skills.

The presentation will focus on the outcomes of the interviews, in particular on how to reach and encourage adults to take advantage of the educational initiatives addressed to them. We will show useful and efficient ways of reaching adults with low basic skills that are practiced in the surveyed organizations and how to successfully involve adult beneficiaries in the projects.

Keywords: adult education, low basic skills, key competences, Upskilling Pathways, educational support 